Tightly Focused Femtosecond Laser Pulses Facilitate Processing of Bulk Glass with Nanojoule Energies

Processing optically transparent materials with laser pulses presents a promising means of micromachining. Focusing a

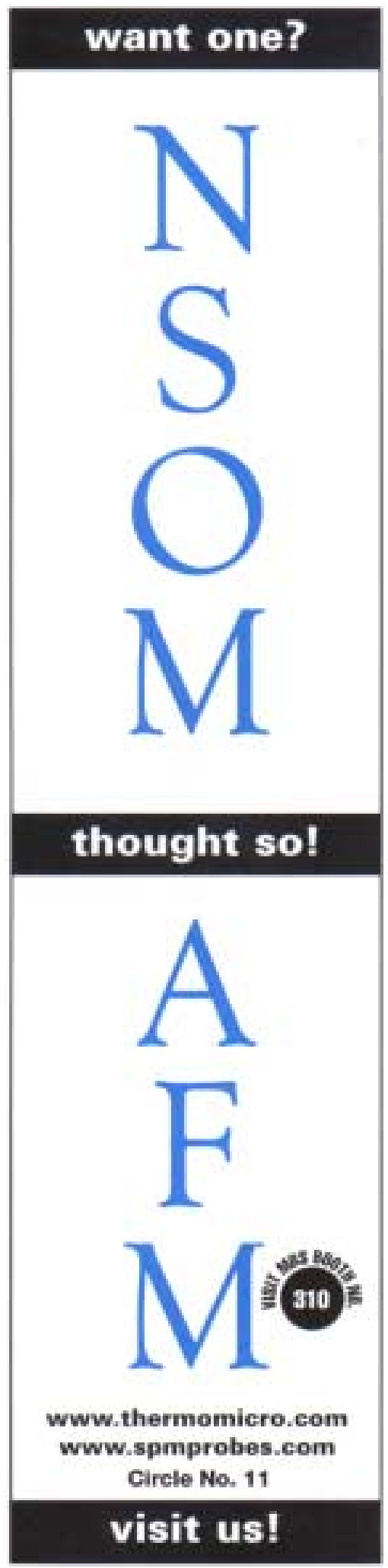

femtosecond laser pulse beneath the surface of a transparent material causes localized and nonlinear energy absorption at the focal volume. This absorption, in turn, leads to optical breakdown and permanent structural changes in the immediate vicinity of the focus while leaving the surface unaffected. To date, however, only amplified, kilohertz repetition-rate femtosecond lasers have had the power necessary to micromachine glass, severely limiting the maximum processing speed and dramatically increasing the cost of the laser system.

As reported in the January 15 issue of Optics Letters, a research group from the Department of Physics at Harvard University has demonstrated that optical breakdown can occur with as little as $5 \mathrm{~nJ}$ of laser pulse energy when a 1.4 NA (numerical aperture) lens is used to focus the pulses. This energy is in the range of modern femtosecond laser oscillators, allowing megahertz repetition-rate unamplified lasers to enter the micromachining arena. The energy threshold for structural alteration as a function of NA was first determined. A 100-fs pump pulse from a Ti:sapphire laser system irradiates a borosilicate glass sample. The laser pulse was focused beneath the surface, using a variety of lenses whose NA ranges from 0.25 to 1.4 . A HeNe laser is used to probe the pumped volume of the sample. When optical breakdown occurs, HeNe light scattered by the structural alteration is detected, allowing the researchers to determine the threshold energy versus NA.

With a single 10-nJ, 100-fs laser pulse focused by a 1.4 NA lens, a cylindrical structure $0.5 \mu \mathrm{m}$ in diameter and $2 \mu \mathrm{m}$ in height was created. Structures with larger diameters were obtained with multiple pulses incident on the same spot in the sample at a high repetition rate. The diameter of these cylinders was found to be proportional to (number of pulses) ${ }^{0.25}$. A third experiment demonstrated that single-mode optical waveguides could be made with the same techniques. Using 5-nJ, 100-fs pulses incident at a $25-\mathrm{MHz}$ repetition rate, an optical waveguide can be written by translating the sample perpendicular to the incident direction of the laser pulse. This waveguide has a near-Gaussian output profile at visible wavelengths. The change in refractive index is about $3 \times 10^{-4}$ from core to rim of the laser-written waveguide.

The straightforwardness of the laser requirements makes structural manipulation of transparent media on the micrometer scale practical. Already the researchers have demonstrated the direct writing of optical waveguides in bulk glass.

JUNE LAU

\section{Composite Materials Produced by Photocatalytic Underpotential Deposition}

The preparation of a $\mathrm{TiO}_{2} / \mathrm{MSe}(\mathrm{M}=\mathrm{Cd}$ and $\mathrm{Pb}$ ) composite material by $\mathrm{TiO}_{2}$-based photocatalysis was recently reported by the research group of Krishnan Rajeshwar, a professor in the Department of Chemistry and Biochemistry at the University of Texas at Arlington. A CdSe or PbSe film was grown on the surfaces of $\mathrm{TiO}_{2}$ particles by a sequential photocatalytic deposition of Se and the metal. The presence of the initial Se layer greatly improved the efficiency of metal deposition. This improvement was attributed to a shift in the redox potential of the metal ions, resulting in the underpotential photodeposition (UPD) of the metal.

As reported in the December 2000 issue of Chemistry of Materials, the deposition of selenium was carried out by UV irradiation of $\mathrm{TiO}_{2}(\mathrm{P}-25)$ particles suspended in an aqueous solution of $\mathrm{Se}^{4+}$. The photoelectrons produced in the $\mathrm{TiO}_{2}$ reduce the $\mathrm{Se}^{4+}$ to form a $\mathrm{Se}^{0}$ layer on the $\mathrm{TiO}_{2}$ surface. After the solution was depleted of $\mathrm{Se}^{4+}$, $\mathrm{Cd}^{2+}$ or $\mathrm{Pb}^{2+}$ was injected into the photoreactor, and the irradiation was repeated. The deposition of $\mathrm{Cd}$ was completed in 100-300 $\mathrm{min}$; the shortest times correspond to optimal Se loading of the $\mathrm{TiO}_{2}$, and longer times correspond to higher- or lower-than-optimal loading. According to Rajeshwar, the slowdown at high loading levels arises because "too much Se means that the Cd flux cannot be maintained." The deposition of $\mathrm{Pb}$ took less than $100 \mathrm{~min}$ at optimal Se coverage. Characterization of the final photodeposition product by $\mathrm{x}$-ray diffraction, x-ray photoelectron spectroscopy, and UV-visible absorption spectroscopy shows the formation of CdSe or PbSe on the $\mathrm{TiO}_{2}$ surface.

The role of the Se layer was determined by attempted photodeposition of $\mathrm{Cd}$ and $\mathrm{Pb}$ onto a bare $\mathrm{TiO}_{2}$ surface. In the absence of $\mathrm{Se}$, the photoreduction of $\mathrm{Pb}^{2+}$ was very slow and that of $\mathrm{Cd}^{2+}$ did not proceed. The researchers attributed the improved deposition rates in the presence of Se to a more positive redox potential of the $\mathrm{M}^{2+}$ /MSe system compared with the $\mathrm{M}^{2+} / \mathrm{M}$ system. This change in redox potential is believed to be the result of the negative free energy of compound formation that exists for the $\mathrm{M}^{2+}$ /MSe system.

According to Rajeshwar, the photocatalytic UPD described in this work is a "simple yet versatile technique for the synthesis of composite materials." Future work in his laboratory will focus on elucidating aspects of the deposition mechanism, and applications of UPD to other semiconductor/template systems.

GREGORY KHITROV 